
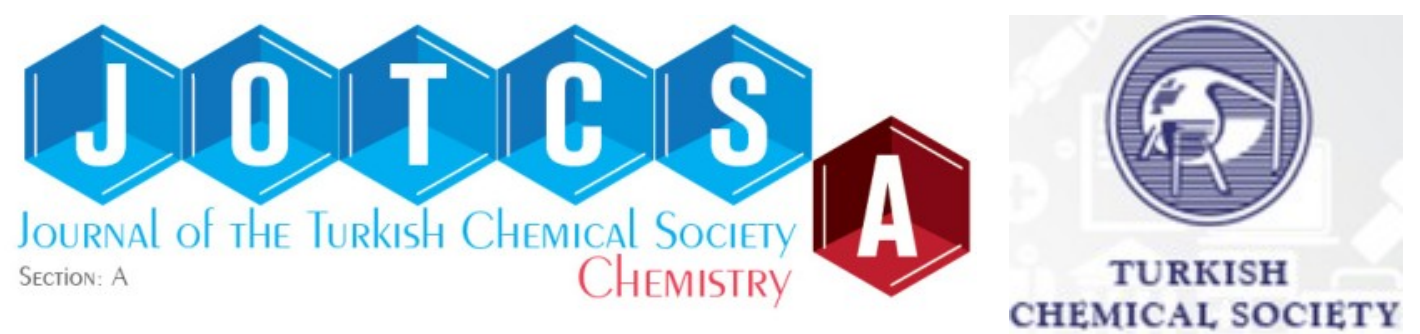

\title{
Non-destructive Detection of Sesame Oil Adulteration by Portable FT- NIR, FT-MIR, and Raman Spectrometers Combined with Chemometrics
}

\author{
Ahmed Menevseoglu ${ }^{1 *}$ \\ ${ }^{1}$ Gumushane University, Faculty of Engineering and Natural Sciences, Department of Food Engineering, \\ Gumushane, 29100, Turkey
}

\begin{abstract}
Edible oils are often adulterated with fixed oils because of their high quality and price. Sesame oil is prone to adulteration due to its high commodity value and popularity. Therefore, a rapid, simple, and non-invasive method to detect adulteration in sesame oil is necessary for quality control purposes. Handheld and portable FT-NIR, FT-MIR, and Raman spectrometers are easy to operate, non-destructive, rapid, and easy to transport for in-situ assessments as well as being cheaper alternatives to traditional instruments. This study aimed to evaluate three different vibrational spectroscopic techniques in detecting sesame oil adulteration with sunflower and canola oil. Sesame oils were adulterated with fixed oils at different concentrations $(0-25 \%)(\mathrm{w} / \mathrm{w})$. Spectra were collected with portable devices and analyzed using Soft Independent Modelling of Class Analogy (SIMCA) to generate a classification model to authenticate pure sesame oil and Partial Least Squares Regression (PLSR) to predict the levels of the adulterant. For confirmation, the fatty acid profile of the oils was determined by gas chromatography (GC). In all three instruments, SIMCA provided distinct clusters for pure sesame oils and adulterated samples with interclass distance (ICD) over 3. Furthermore, FT-NIR and FT-MIR showed excellent performance in predicting adulterant levels with $r_{\text {val }}>0.96$. Specifically, the FT-MIR unit provided more precise classification and PLSR prediction models over FT-NIR and Raman units. Still, all the units can be used as an alternative method to traditional methods such as GC, GC-MS, etc. These units showed great potential for in-situ surveillance to detect sesame oil adulterations.
\end{abstract}

Keywords: Sesame oil, adulteration, portable devices, NIR, MIR, Raman.

Submitted: May 21, 2021. Accepted: July 08, 2021.

Cite this: Menevseoglu A. Non-destructive Detection of Sesame Oil Adulteration by Portable FT-NIR, FTMIR, and Raman Spectrometers Combined with Chemometrics. JOTCSA. 2021;8(3):775-86.

DOI: https://doi.org/10.18596/jotcsa.940424.

*Corresponding author. E-mail: amenevseoglu@gumushane.edu.tr, Tel.: +90-456-233-10-00/1849.

\section{INTRODUCTION}

Sesame oil is a seed oil extracted from Sesamum indicum L. Because it provides many health benefits and contains antioxidants, polyunsaturated fatty acids, tocopherols, sesamin and sesamol, which are cardioprotective functional components, it is becoming more popular and demanded worldwide $(1,2)$. Sesame oil consists of up to $48 \%$ linoleic acid, $43 \%$ oleic acid, $12 \%$ palmitic acid, and 7\% stearic acid (3). Sesame oil is used in foods as a flavor enhancer, in shortening, cosmetics, and pharmaceuticals $(4,5)$.
China, Myanmar, India, and Nigeria are the leading countries for sesame oil production, followed by Japan (6). The largest sesame oil producer, China, had an annual production of 302,354 tons $(29 \%)$, followed by Myanmar with 168,100 tons $(16 \%)$, India with 87,200 tons $(8 \%)$, Nigeria with 54,000 tons $(5 \%)$, and Japan with 52,700 tons $(5 \%)$ in 2018 (6). Sesame oil is a premium oil, therefore an expensive oil, which increases the concern of authentication. Sunflower, canola, maize, and soybean oils are the most common oils used for adulterations in sesame oil (7). 
Conventional analytic techniques such as GC, HPLC, NMR, and IMS have focused on specific marker compounds in the pure oils (4). These traditional methods focus on targeted approaches that pre-define the chemical marker for their identification and quantification; however, those can be failed to detect unexpected adulterants that cannot be determined through the selected method (8). Although these methods susceptible and accurate, they require transportation of samples to the facilities where analysis is carried, are invasive, expensive, time-consuming, and require high-cost instrumentation and maintenance and complex sample preparation (9). Vibrational spectroscopy techniques (VST) combined with multivariate data analysis can be used as an alternative to the conventional techniques because VST can provide rapid, precise, and sensitive chemical information of the samples. Besides, a non-targeted approach using VST focuses on all chemical compounds without knowing the sample's chemical structure, which could then be compared with the pure sample's fingerprint profile (10). The performance of VST on the detection of adulteration in the edible oils has been evaluated in the literature, including olive oil (8), avocado oil (11), and sesame oil (12). With the advancement of microelectromechanical systems, fibers, sensors, optical parts, and detectors have been assisted the miniaturization of vibrational spectroscopy units. These units can provide in-situ analysis, real-time assessments, rapid and cost-efficient results because there is no need to transport the samples and convenience to the food industry and food control agencies (13).

This study aimed to evaluate the performance of three different vibrational spectroscopy techniques and portable devices on the authentication of sesame oil.

\section{MATERIALS and METHODS}

\section{Materials}

Sesame oils (32 different commercial products), sunflower oils (6 different commercial products), canola oils (6 different commercial products) were purchased from various local markets in Istanbul, Turkey, and Columbus, $\mathrm{OH}$, USA. Sunflower and canola oil were selected because both oils were cheaper than sesame oil and could be used as adulterants in sesame oil. Samples were stored at $4{ }^{\circ} \mathrm{C}$ until further analysis to prevent any oxidative changes in the oil. One of the pure sesame oils was chosen randomly, and it was adulterated with 5 , $10,15,20$, and $25 \%(w / w)$ sunflower oil and canola oil, separately. Adulteration with adulterant levels lower than $10 \%$ may not be feasible economically; therefore, it may limit the use in food fraud practices, and high adulterant levels may be easily detected by sensory without any instrument used. Therefore, a 5-25\% range was selected to imitate realistic and practical applications in the adulteration of oils (14).

\section{Methods}

NIR

The NIR of the oil samples were collected using micro-NeoSpectra (Si-Ware Systems, Cairo, Egypt) (Figure 1), a compact Fourier Transform NearInfrared (FT-NIR) sensor equipped with a singlechip Michelson interferometer with monolithic Opto-electro-mechanical structure with a single uncooled indium-gallium-arsenide (InGaAs) photodetector. A total of $50 \mu \mathrm{L}$ of oil sample were deposited on the unit's sensor, and the spectra were collected at the range of $7400-3920 \mathrm{~cm}^{-1}$ in absorbance mode and with a $16 \mathrm{~cm}^{-1}$ resolution. Samples were scanned for 15 seconds to increase the signal-to-noise ratio. Spectral data collection was carried out in duplicate.

\section{MIR}

A total of $50 \mu \mathrm{L}$ of the oil sample was placed on the IR crystal. The FT-IR 5500 (Agilent Technologies Inc., Santa Clara, CA) (Fig. 1) spectrometer equipped with a temperature-controlled 5-bounce ZnSe crystal set to $40{ }^{\circ} \mathrm{C}$. The spectra were collected at $4 \mathrm{~cm}^{-1}$ resolution over a range from 4000 to $650 \mathrm{~cm}^{-1}$, and an interferogram of 64 scans co-added to increase the signal-to-noise ratio. Spectral data collection was carried out in duplicate.

\section{Raman}

Three milliliters of oil sample were placed in a 10$\mathrm{mm}$ light path quartz cuvette (Hellma Analytics, Mulheim, Germany), and the Raman spectra were collected using a WP 1064 compact portable Raman spectrometer (Wasatch Photonics, Durham, NC, USA) (Figure 1). The Raman unit was equipped with an Indium Gallium Arsenide (InGaAs) detector and a laser source operating at $1064 \mathrm{~nm}$. The spectra were collected from 1855 to $200 \mathrm{~cm}^{-1}$ with a resolution of $4 \mathrm{~cm}^{-1}$, and 3 scans were co-added to improve the signal-to-noise ratio of the spectrum with an integration time of 3000 ms. A background spectrum was acquired in between every sample to eliminate the environmental variations. Spectral data were displayed in terms of scattered light by the sample and viewed using EnlightenTM software (Wasatch Photonics, Durham, NC, USA). Spectral data collection was carried out in duplicate.

\section{GC (Reference method)}

The fatty acid profile was determined using a fatty acid methyl ester (FAME) procedure (15) with slight modifications. A total of $100 \mu \mathrm{L}$ oil sample was dissolved in $1 \mathrm{~mL}$ of $\mathrm{n}$-hexane in a microcentrifuge tube; after adding $20 \mu \mathrm{L}$ of $2 \mathrm{~N}$ potassium hydroxide in methanol, the mixture was 
vortexed for a minute. The microcentrifuge tube set aside for the phase separation, and a $750 \mu \mathrm{L}$ of an aliquot from the upper part was removed into another microcentrifuge tube with a pinch of anhydrous sodium sulfate. The tube was centrifuged at $13.2 \mathrm{rpm}$ for $5 \mathrm{~min}$, and the upper part $(\sim 500 \mu \mathrm{L})$ was transferred into a borosilicate glass vial. Samples were analyzed using an Agilent 6890 (Santa Clara, CA, USA) GC, with a flame ionization detector (FID) and an HP G1513A autosampler and a tray. Fatty acids were eluted through an HP-88 $100 \mathrm{~m} \times 0.25 \mathrm{~mm} \times 0.2 \mu \mathrm{m}$ column (Agilent, Santa Clara, CA, USA), and helium was used as a carrier gas. A total of $1 \mu \mathrm{L}$ of the sample was injected with a split ratio of $20: 1$. The oven temperature was set at $110^{\circ} \mathrm{C}$ for $1 \mathrm{~min}$, then increased to $220^{\circ} \mathrm{C}\left(5^{\circ} \mathrm{C} / \mathrm{min}\right)$ and held for $15 \mathrm{~min}$. The injector temperature was $220^{\circ} \mathrm{C}$, and the detector temperature was $250{ }^{\circ} \mathrm{C}$. The identification of the fatty acids was achieved by comparing each peak's retention time against the reference standards (Supelco $\AA 37$ Component FAME Mix, Sigma Aldrich, St. Louis, MO, USA). GC analyses for each sample were carried out in duplicate.

\section{Data Analysis}

A multivariate data analysis software (Pirouette version 4.5, Infometrix Inc., Bothell, WA, USA) was used to analyze the spectral data.

\section{SIMCA}

A supervised pattern recognition technique relies on a confidence region for each class after PCA is applied. Observations are projected in each PC that decides if the observation belongs to that class or not. Because the spectra are complex, SIMCA is required for obtaining meaningful information of the data matrix (16). More information can be found in the literature (17-19). SIMCA was used to determine the pure samples and adulterated sesame oils. Known classes (sesame, sunflower, and canola oils) were used to create a classification algorithm to identify the correct classes ( $80 \%$ of the total samples). Then, the validation data set ( $20 \%$ of the samples that were not used in the classification model) was informed to evaluate the model. SIMCA was evaluated based on threedimension class projection, misclassification (if the sample was predicted in the correct class), and interclass distance (ICD).

\section{PLSR}

Partial Least Square Regression (PLSR) was used to evaluate the fitness of the model using the standard error of cross-validation (SECV), standard error of prediction (SEP), coefficient of correlation (r), and outlier diagnostics. PLSR is one of the most used multivariate data analyses used for correlated, noisy, and multi-X variables. For example, data obtained from FT-NIR, FT-MIR, and Raman can have thousands of data points ( $X$ variable, i.e., FT-MIR - from $3500 \mathrm{~cm}^{-1}$ to $800 \mathrm{~cm}^{-}$ $\left.{ }^{1}\right)$. PLSR includes the PCA features where thousands of variables can be compressed into few latent variables called factors, predictors, or components. Detailed information for PLSR can be found in the literature (20-23). Samples with large residuals indicating the samples with structure do not fit in the model, and high leverage indicates the sample or variable have very much impact on the calibration model were considered as outliers (not shown in the data).

\section{RESULTS AND DISCUSSION}

\section{SIMCA Results - FT-NIR Spectra}

Due to NIR spectra's complexity, and to make the interpretation more straightforward, the spectra were mean-centered, second derivative, and smoothly transformed before the SIMCA analysis to obtain better spectral characteristics (with a second-order poly-nominal filter with a 19-point window). Even though many pre-processing options were tried, the best results were obtained using a 19-point window (i.e., 21, 25, 35-point). SIMCA was used to generate a classification algorithm for the oils. Figure 2A shows the SIMCA 3D projection to classify sesame oil, sunflower oil, and canola oil. Based on the SIMCA, all oil samples were clustered very distinctly ( $I C D>19)$. Figure $2 B$ shows the classification's discriminating power, which expresses the variables (wavenumber $\left(\mathrm{cm}^{-}\right.$ $\left.{ }^{1}\right)$ ) responsible for the classification. Peak identifications were made based on the literature $(24,25)$. Around $4386 \mathrm{~cm}^{-1}$, the combination of $\mathrm{C}-\mathrm{H}$ stretching vibration can be observed. The peak at $4876 \mathrm{~cm}^{-1}$ is associated with the $\mathrm{C}-\mathrm{H}$ vibration of cis-unsaturation. Lastly, the peak at $5660 \mathrm{~cm}^{-1}$ is related to the first overtone of the $\mathrm{C}-\mathrm{H}$ stretching vibration of chemical groups (i.e., methylene). Figure 2C shows the FT-NIR prediction. The prediction model indicated that adulterated samples were clustered from pure samples. Moreover, two out of eight validation samples (not used in calibration set) did not fit in the model, and GC data confirmed that those samples' fatty acid profile was different from pure samples. Only one sample was misclassified when comparing FT-MIR and Raman results. Figure 2D shows the score plot of the model, indicating that using two factors was enough to represent the model with $\sim 97 \%$. Overall, a miniaturized FT-NIR spectrometer combined with SIMCA showed that FT-NIR could be an alternative method for detecting sesame oil adulteration with cheap vegetable oils. 

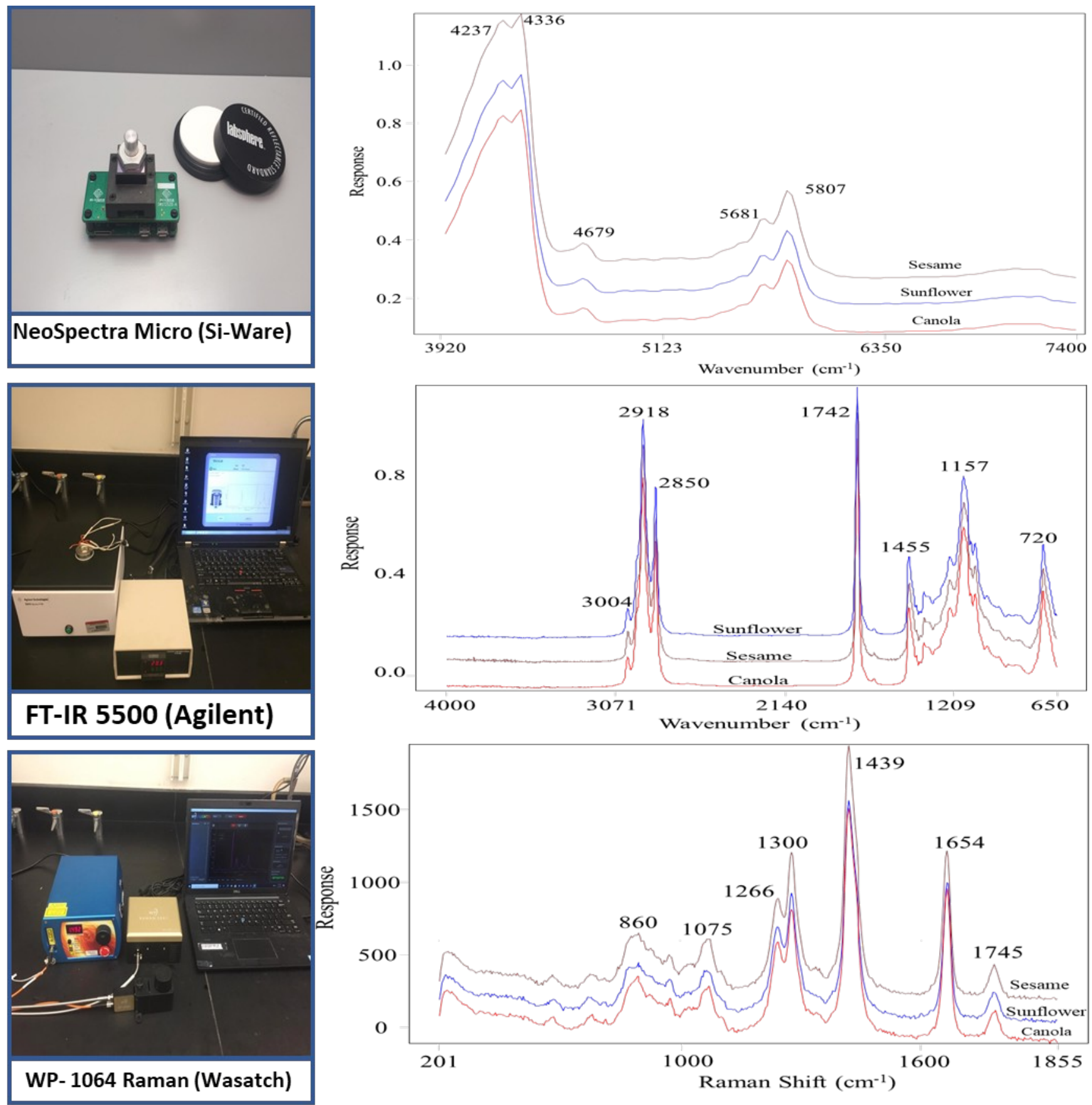

Figure 1: Commercial portable and handheld FT-NIR, FT-MIR and Raman spectrometers, and the spectra of oils obtained from them. 

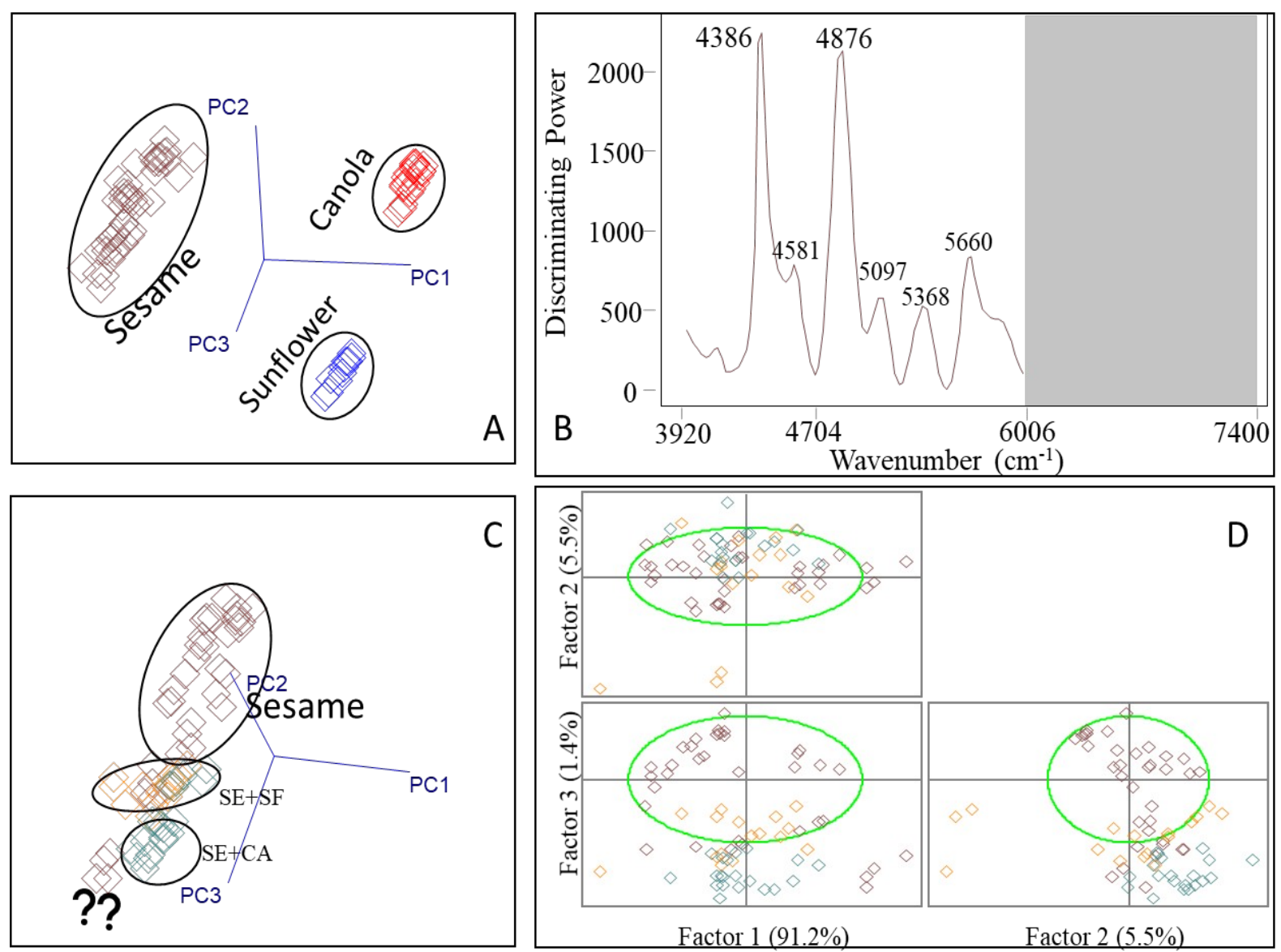

Figure 2: SIMCA 3D projection obtained from FT-NIR spectral data (2A), discriminating power obtained from SIMCA (2B), Validation model of SIMCA (2C), score plot for validation model (2D). SE: sesame oil; SF: sunflower oil; CA: canola oil; ? mark: suspected sample.

\section{SIMCA Results - FT-MIR Spectra}

Before the SIMCA analysis, the spectra were mean-centered, local-scoped, first-derivative, normalized, and smooth-transformed (19-point window) for improving spectral characteristics. Figure 3A shows SIMCA 3D projection obtained from FT-MIR spectral data. The samples were clustered distinctly with ICD $>10$. Based on the ICD value, FT-NIR was slightly better than FT-MIR. Figure 3B shows the discriminating power, and the peaks assignment was made based on previous studies $(26,27)$. The peaks around in the range of 3024 - $2864 \mathrm{~cm}^{-1}$ associated with $\mathrm{C}-\mathrm{H}$ stretching of methyl and methylene groups $\left(\mathrm{CH}_{2}\right.$ symmetric and $\mathrm{CH}_{3}$ asymmetric stretching), $1836-1709 \mathrm{~cm}^{-1}$ related to $-\mathrm{C}=\mathrm{O}$ ester stretching vibration ( $\mathrm{C}-\mathrm{O}$ stretching esters of fatty acids), $1450-1377 \mathrm{~cm}^{-1}$ associated with $\mathrm{C}-\mathrm{H}$ bending (symmetrical and scissoring) vibrations of $\mathrm{CH}_{2}$ and $\mathrm{CH}_{3}$ groups.
Although the oil spectral profile seems similar, triglyceride composition would impact the band intensities and slight shifts in the wavenumbers for functional groups. Figure $3 \mathrm{C}$ shows the prediction model. Similar to the FT-NIR results, adulterated samples were clustered very distinctly. Besides, three out of eight external validation samples did not fit in the model, and GC results were confirmed the fatty acid profile of those samples were different than pure sesame oils. SIMCA results indicated that even with low adulterant levels (5\%) could be detected by FT-MIR spectrometer. Figure 3D shows the score plot of the SIMCA validation model obtained from FT-MIR spectral data indicating that using three factors was enough to represent the prediction model with $\sim 88 \%$ of all variables. Overall, portable FT-MIR unit showed great performance in detecting sunflower and canola oil adulterations in sesame oil. 

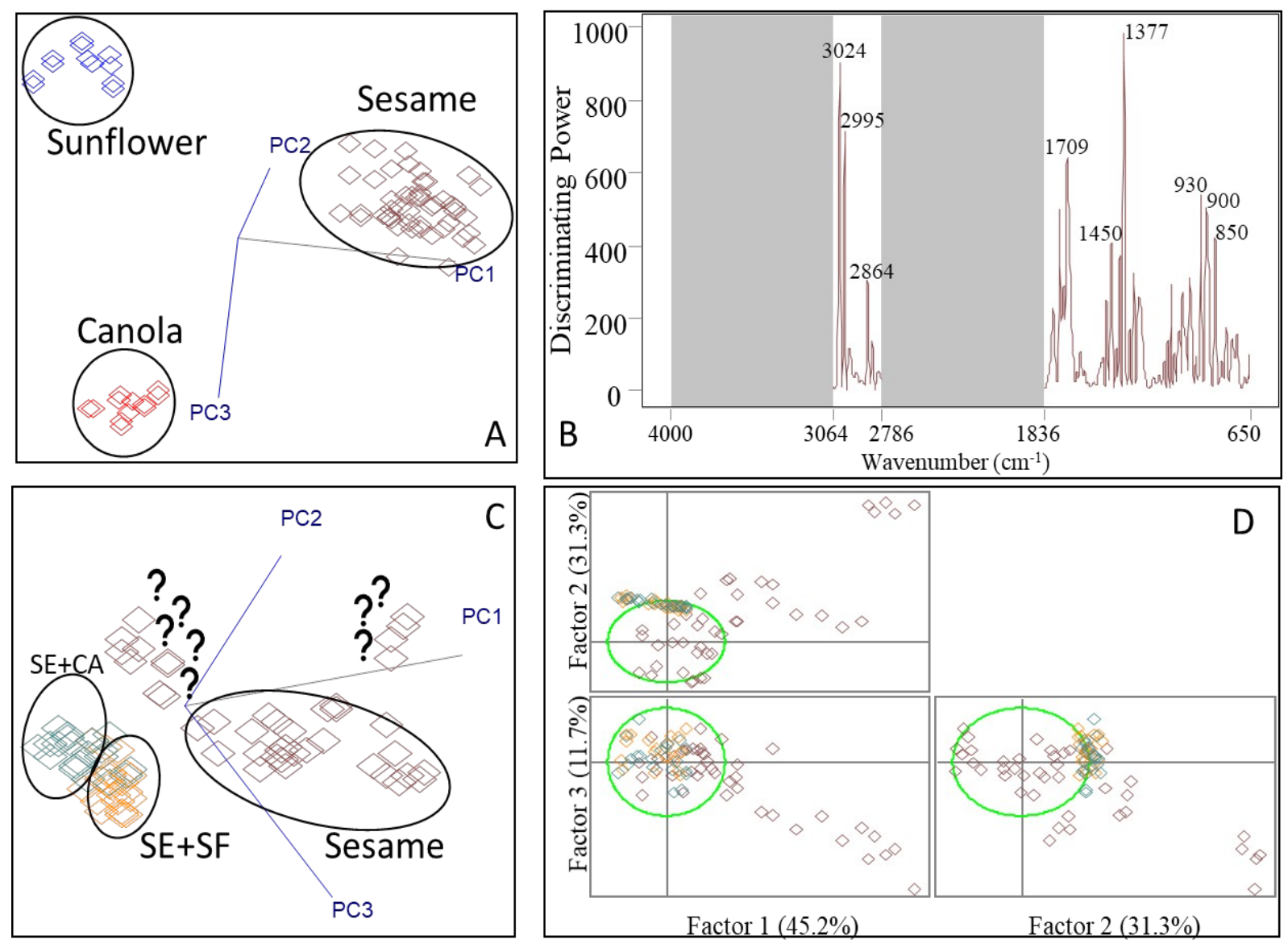

Figure 3 SIMCA 3D projection obtained from FT-MIR spectral data (3A), discriminating power obtained from SIMCA (3B), Validation model of SIMCA (3C), score plot for validation model (3D). SE: sesame oil; SF: sunflower oil; CA: canola oil; ? mark: suspected sample.

\section{SIMCA Results - Raman Spectra}

Similar to previously mentioned techniques, spectral data were mean-centered, locally scoped, second-derivative, and smooth-transformed (19point window). Figure $4 \mathrm{~A}$ shows the classification of three oils. Based on the spectral data, all three oils were clustered distinctly with ICD $>5$. The classification obtained from Raman spectral data was slightly worse than both FT-MIR and FT-NIR data based on the ICD value. A possible explanation for this result would be the due less scanning region (wavenumber) in our Raman spectrometer. For instance, FT-MIR covers 3064 $2786 \mathrm{~cm}^{-1}$, which region associated with $\mathrm{CH}_{2}$ and $\mathrm{CH}_{3}$; however, Raman covers up to $1855 \mathrm{~cm}^{-1}$ in our unit. Figure 4B shows the discriminating power for classification. $1445 \mathrm{~cm}^{-1}$, associated with $-\mathrm{CH}_{2}$ bending vibrational mode, is the most powerful band for classification. $1291 \mathrm{~cm}^{-1}$ (twisting $-\mathrm{CH}_{2}$ ), $1640 \mathrm{~cm}^{-1}$ and $1657 \mathrm{~cm}^{-1}$ (stretching cis-R$\mathrm{HC}=\mathrm{CH}-\mathrm{R}$ ) were also powerful bands for classification. The peaks assignments were made based on the literature (28). Figure 4C shows the validation model. Similar to FT-MIR unit, Raman spectrometer detected the adulterated samples, and three suspected samples which then confirmed by GC-FID, and those were clustered distinctly. SIMCA results indicated that a Raman spectrometer could detect as low as $5 \%$ adulteration in sesame oil. Figure 4D shows the score plot for validation model indicated three factors were enough to represent the prediction model with $\sim 93 \%$ of all variables. Overall, the portable Raman spectrometer showed good performance in detecting sesame oil adulteration with sunflower and canola oil. 

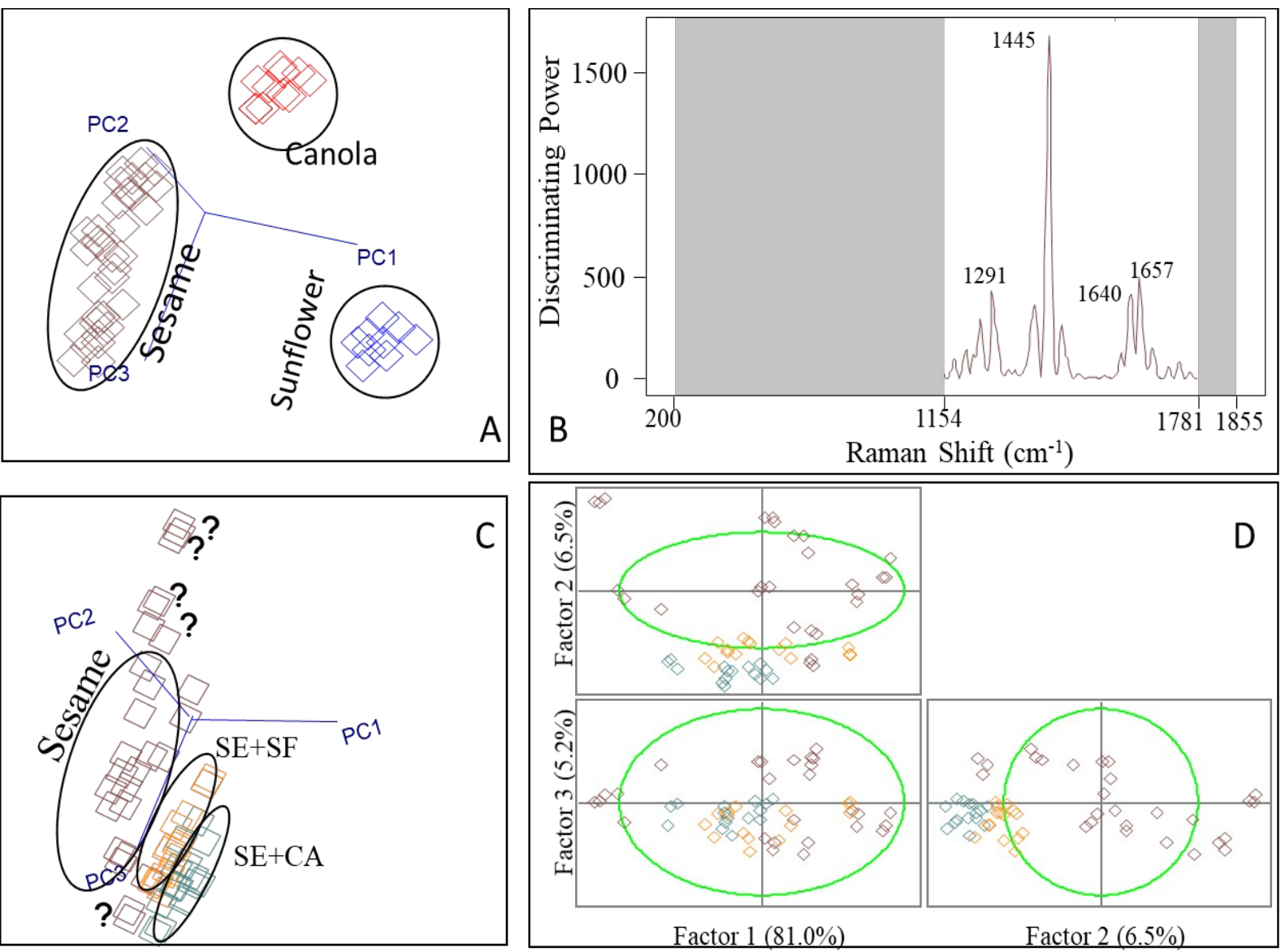

Figure 4 SIMCA 3D projection obtained from Raman spectral data (4A), discriminating power obtained from SIMCA (4B), Validation model of SIMCA (4C), score plot for validation model (4D). SE: sesame oil; SF: sunflower oil; CA: canola oil; ? mark: suspected sample.

\section{PLSR Results - FT-NIR, FT-MIR, and Raman Spectra}

Table 1 shows the prediction performance of three vibrational spectroscopy units for predicting adulterant levels in sesame oil. The optimum factors for predicting models were between four to six. Although the performance would be slightly better if more factors were used, using more factors would include noise or irrelevant components that would cause overfitting the model. Likewise, using fewer factors than optimum number would cause underfitting associated with less variance than needed (29). PLSR models of the oils yielded high SECV, high SEP, $r_{\text {cal }}$, and $r_{\text {val }}$ values, indicating the accuracy of the prediction models. Correlation coefficient value $(r)$ is accepted as an excellent prediction over 0.90 , and suitable prediction over 0.80 (30). FT-NIR, FT-MIR, and Raman units showed excellent performance in predicting adulterant levels $\left(r_{\text {val }}>0.90\right)$. Portable FT-MIR unit showed superior performance over handheld FT-NIR and portable Raman units since it provided lower SEP, and higher $r_{\text {val }}$. 



Figure 5 Partial Least Square Regression (PLSR) calibration and validation plots for sunflower oil levels (5A), canola oil (5B) obtained from FT-NIR unit, 5C and 5D obtained from FT-MIR unit, 5E and 5F obtained from Raman unit. Empty squares: calibration model; filled squares: validation model. 
Table 1: Statistical performance of the prediction models developed using FT-NIR, FT-MIR, and Raman spectrometers for adulteration levels in sesame oil.

\begin{tabular}{|c|c|c|c|c|c|c|c|c|c|c|}
\hline \multirow{2}{*}{ Unit } & \multirow{2}{*}{ Parameter } & \multicolumn{5}{|c|}{ Calibration model } & \multicolumn{4}{|c|}{ Validation model } \\
\hline & & Range $^{a}$ & $\mathbf{N}^{\mathbf{b}}$ & $F^{c}$ & SECV $^{d}$ & $\mathbf{r c a l}{ }^{\mathbf{e}}$ & Range & $\mathbf{N}$ & SEPf & $r_{\text {val }}{ }^{g}$ \\
\hline \multirow{2}{*}{ FT-NIR } & SFO & $0-25$ & 18 & 5 & 1.17 & 0.994 & $5.0-20.0$ & 12 & 2.03 & 0.976 \\
\hline & CAO & $0-25$ & 18 & 6 & 1.76 & 0.985 & $5.0-20.0$ & 12 & 2.28 & 0.969 \\
\hline \multirow[t]{2}{*}{ FT-MIR } & SFO & $0-25$ & 18 & 6 & 1.41 & 0.990 & $5.0-20.0$ & 12 & 1.82 & 0.978 \\
\hline & CAO & $0-25$ & 18 & 4 & 0.95 & 0.996 & $5.0-20.0$ & 12 & 1.36 & 0.988 \\
\hline \multirow[t]{2}{*}{ Raman } & SFO & $0-25$ & 18 & 5 & 2.83 & 0.937 & $5.0-20.0$ & 12 & 3.23 & 0.898 \\
\hline & $\mathrm{CAO}$ & $0-25$ & 18 & 4 & 2.87 & 0.924 & $5.0-20.0$ & 12 & 3.26 & 0.909 \\
\hline
\end{tabular}

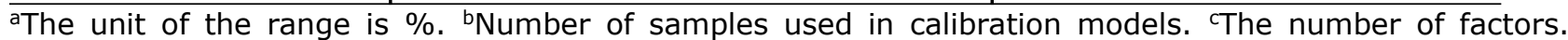

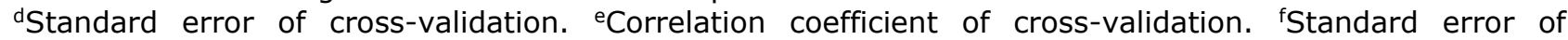
prediction. ${ }^{9}$ Correlation coefficient of prediction for external validation. SFO: Sunflower oil. CAO: Canola oil.

\section{CONCLUSIONS}

In this study, sesame oil adulteration with sunflower and canola oil was aimed to be determined by portable FT-NIR, FT-MIR, and Raman spectrometers. Developed SIMCA and PLSR models showed that sesame oil adulteration could be detected rapidly, non-destructively, and reliably by using portable vibrational spectroscopy units. Based on portable units' and GC results, three out of 32 samples were suspected as being adulterated. FT-MIR spectrometer showed slightly better performance than FT-NIR, superior performance than Raman spectrometers. These techniques may detect sunflower and canola oil adulterations in sesame oil as low as $5 \%$. These portable units can provide government agencies and the oil industry an alternative method to the traditional methods.

\section{FUNDING}

This research received no external funding.

\section{CONFLICT OF INTEREST}

The author declares no conflict of interest.

\section{ACKNOWLEDGMENTS}

The author would like to thank Prof. Luis E. Rodriguez-Saona and Didem Peren Aykas, PhD (The Ohio State University, Department of Food Science and Technology) for their technical support rendered during this study.

\section{REFERENCES}

1. Ozulku G, Yildirim RM, Toker OS, Karasu S, Durak MZ. Rapid detection of adulteration of cold pressed sesame oil adultered with hazelnut, canola, and sunflower oils using ATR-FTIR spectroscopy combined with chemometric. Food Control. 2017 Dec;82:212-6. DOI:

https://doi.org/10.1016/j.foodcont.2017.06.034.

2. Wang R, Liu K, Wang X, Tan M. Detection of Sesame Oil Adulteration Using Low-Field Nuclear Magnetic Resonance and Chemometrics.

International Journal of Food Engineering. $2019 \mathrm{Jul}$ 26;15(7):20180349. DOI:

https://doi.org/10.1515/ijfe-2018-0349.

3. Gharby S, Harhar H, Bouzoubaa Z, Asdadi A, El Yadini A, Charrouf Z. Chemical characterization and oxidative stability of seeds and oil of sesame grown in Morocco. Journal of the Saudi Society of Agricultural Sciences. 2017 Apr;16(2):105-11. DOI: https://doi.org/10.1016/j.jssas.2015.03.004.

4. Zhang L, Shuai Q, Li P, Zhang Q, Ma F, Zhang $W$, et al. Ion mobility spectrometry fingerprints: $A$ rapid detection technology for adulteration of sesame oil. Food Chemistry. 2016 Feb;192:60-6. DOI:

https://doi.org/10.1016/j.foodchem.2015.06.096.

5. Warra A. Sesame (Sesamum indicum L.) Seed Oil Methods of Extraction and its Prospects in Cosmetic Industry: A Review. Bayero J Pure App Sci. 2012 Apr 6;4(2):164-8. DOI:

https://doi.org/10.4314/bajopas.v4i2.33.

6. FAO. FAOSTAT online statistical service [Internet]. FAO; 2018. Available from: http://faostat.fao.org/.

7. Seo H-Y, Ha J, Shin D-B, Shim S-L, No K-M, Kim $\mathrm{K}-\mathrm{S}$, et al. Detection of Corn Oil in Adulterated Sesame Oil by Chromatography and Carbon Isotope Analysis. J Am Oil Chem Soc. 2010 Jun;87(6):621-6. DOI:

https://doi.org/10.1007/s11746-010-1545-6. 
8. Aykas DP, Karaman AD, Keser B, RodriguezSaona L. Non-Targeted Authentication Approach for Extra Virgin Olive Oil. Foods. 2020 Feb 20;9(2):221. DOI:

https://doi.org/10.3390/foods9020221.

9. Subramanian A, Alvarez VB, Harper WJ, Rodriguez-Saona LE. Monitoring amino acids, organic acids, and ripening changes in Cheddar cheese using Fourier-transform infrared spectroscopy. International Dairy Journal. 2011 Jun;21(6):434-40. DOI: https://doi.org/10.1016/j.idairyj.2010.12.012.

10. Tengstrand E, Rosén J, Hellenäs K-E, Åberg $\mathrm{KM}$. A concept study on non-targeted screening for chemical contaminants in food using liquid chromatography-mass spectrometry in combination with a metabolomics approach. Anal Bioanal Chem. 2013 Feb;405(4):1237-43. DOI: https://doi.org/10.1007/s00216-012-6506-5.

11. Quiñones-Islas N, Meza-Márquez OG, OsorioRevilla G, Gallardo-Velazquez T. Detection of adulterants in avocado oil by Mid-FTIR spectroscopy and multivariate analysis. Food Research International. 2013 Apr;51(1):148-54. DOI:

https://doi.org/10.1016/j.foodres.2012.11.037.

12. Chen $\mathrm{H}$, Lin $\mathrm{Z}$, Tan C. Fast quantitative detection of sesame oil adulteration by nearinfrared spectroscopy and chemometric models. Vibrational Spectroscopy. 2018 Nov;99:178-83. DOI:

https://doi.org/10.1016/j.vibspec.2018.10.003.

13. Rodriguez-Saona L, Aykas DP, Borba KR, Urtubia A. Miniaturization of optical sensors and their potential for high-throughput screening of foods. Current Opinion in Food Science. 2020 Feb;31:136-50. DOI:

https://doi.org/10.1016/j.cofs.2020.04.008.

14. Miaw CSW, Sena MM, Souza SVC de, Ruisanchez I, Callao MP. Variable selection for multivariate classification aiming to detect individual adulterants and their blends in grape nectars. Talanta. 2018 Dec;190:55-61. DOI: https://doi.org/10.1016/j.talanta.2018.07.078.

15. Ichihara K, Shibahara A, Yamamoto K, Nakayama T. An improved method for rapid analysis of the fatty acids of glycerolipids. Lipids. 1996 May;31(5):535-9. DOI:

https://doi.org/10.1007/BF02522648.

16. De Maesschalck R, Candolfi A, Massart DL, Heuerding S. Decision criteria for soft independent modelling of class analogy applied to near infrared data. Chemometrics and Intelligent Laboratory
Systems. 1999 Apr;47(1):65-77. DOI: https://doi.org/10.1016/S0169-7439(98)00159-2.

17. Wold S. Pattern recognition by means of disjoint principal components models. Pattern Recognition. 1976 Jul;8(3):127-39. DOI: https://doi.org/10.1016/0031-3203(76)90014-5.

18. Lavine BK. Clustering and Classification of Analytical Data. In: Meyers RA, editor.

Encyclopedia of Analytical Chemistry [Internet] Chichester, UK: John Wiley \& Sons, Ltd; 2000 [cited 2021 Jul 17]. p. a5204. Available from: https://onlinelibrary.wiley.com/doi/10.1002/97804 70027318.a5204.

19. Ballabio D, Todeschini R. Infrared Spectroscopy for Food Quality Analysis and Control Multivariate Classification for Qualitative Analysis. In: Sun D-W, editor. Infrared Spectroscopy for Food Quality Analysis and Control. 1st edition. Burlington, MA: Elsevier; 2009. p. 83-104. ISBN: 978-0-12374136-3.

20. Haaland DM, Thomas EV. Partial least-squares methods for spectral analyses. 1. Relation to other quantitative calibration methods and the extraction of qualitative information. Anal Chem. 1988 Jun 1;60(11):1193-202. DOI: https://doi.org/10.1021/ac00162a020.

21. Jong SD. PLS fits closer than PCR. J Chemometrics. 1993 Nov;7(6):551-7. DOI: https://doi.org/10.1515/jpme.1998.26.4.325.

22. Brereton RG. Introduction to multivariate calibration in analytical chemistry. Analyst. 2000;125(11):2125-54. DOI: https://doi.org/10.1039/b003805i.

23. Wold S, Sjöström M, Eriksson L. PLSregression: a basic tool of chemometrics. Chemometrics and Intelligent Laboratory Systems. 2001 Oct; 58(2):109-30. DOI:

https://doi.org/10.1016/S0169-7439(01)00155-1.

24. Hourant $P$, Baeten V, Morales MT, Meurens M, Aparicio R. Oil and Fat Classification by Selected Bands of Near-Infrared Spectroscopy. Appl Spectrosc. 2000 Aug;54(8):1168-74. URL: http://as.osa.org/abstract.cfm?URI=as-54-8-1168.

25. Yang $H$, Irudayaraj J, Paradkar $M$. Discriminant analysis of edible oils and fats by FTIR, FT-NIR and FT-Raman spectroscopy. Food Chemistry. 2005 Nov; 93(1):25-32. DOI:

https://doi.org/10.1016/j.foodchem.2004.08.039.

26. Aykas DP, Rodriguez-Saona LE. Assessing potato chip oil quality using a portable infrared spectrometer combined with pattern recognition 
analysis. Anal Methods. 2016;8(4):731-41. DOI: https://doi.org/10.1039/C5AY02387D.

27. Rodriguez-Saona LE, Giusti MM, Shotts M. Advances in Infrared Spectroscopy for Food Authenticity Testing. In: Advances in Food Authenticity Testing [Internet]. Elsevier; 2016 [cited 2021 Jul 17]. p. 71-116. Available from: https://linkinghub.elsevier.com/retrieve/pii/B9780 $\underline{081002209000047}$

28. Covaciu F-D, Berghian-Grosan C, Feher I, Magdas DA. Edible Oils Differentiation Based on the Determination of Fatty Acids Profile and Raman Spectroscopy-A Case Study. Applied Sciences. 2020 Nov 24;10(23):8347. DOI:

https://doi.org/10.3390/app10238347.

29. Abdi H. Partial least squares regression and projection on latent structure regression (PLS Regression). WIREs Comp Stat. 2010 Jan;2(1):97106. DOI: https://doi.org/10.1002/wics.51.

30. Urbanocuadrado $M$, Luquedecastro $M$, Perezjuan P, Gomeznieto M. Comparison and joint use of near infrared spectroscopy and Fourier transform mid infrared spectroscopy for the determination of wine parameters. Talanta. 2005 Mar 31;66(1):218-24. DOI:

https://doi.org/10.1016/j.talanta.2004.11.011. 
Menevseoglu A. JOTCSA. 2021; 8(3): 775-786.

RESEARCH ARTICLE 